\title{
Two-flavor adjoint QCD
}

\author{
Mohamed M. Anber \\ Department of Physics, Lewis \& Clark College, Portland, Oregon 97219, USA \\ Erich Poppitz ${ }^{\dagger}$ \\ Department of Physics, University of Toronto, Toronto, Ontario M5S 1A7, Canada
}

(Received 8 June 2018; published 28 August 2018)

\begin{abstract}
We study four-dimensional $S U(2)$ Yang-Mills theory with two massless adjoint Weyl fermions. When compactified on a spatial circle of size $L$ much smaller than the strong-coupling scale, this theory can be solved by weak-coupling nonperturbative semiclassical methods. We study the possible realizations of symmetries in the $\mathbb{R}^{4}$ limit and find that all continuous and discrete 0 -form and 1-form 't Hooft anomalymatching conditions are saturated by a symmetry realization and massless spectrum identical to that found in the small- $L$ limit, with only a single massless flavor-doublet fermion in the infrared. This observation raises the possibility that the class of theories which undergo no phase transition between the analytically solvable small-size circle and strongly coupled infinite-size circle is larger than previously thought and offers new challenges for lattice studies.
\end{abstract}

DOI: 10.1103/PhysRevD.98.034026

\section{INTRODUCTION}

The solution of general four-dimensional strongly coupled gauge theories remains an elusive goal. Assuming large amounts of symmetry, e.g., extended supersymmetry, helps make progress, but the resulting theories are often quite distinct from the ones describing the known interactions of elementary particles, or from various conjectured extensions of the Standard Model. Putting gauge theories on a space-time lattice has proven tremendously useful but is a subject of severe technical (or conceptual) difficulties, especially for theories with global (or gauged) chiral symmetries.

Thus, any new handle to study the nonperturbative behavior of four-dimensional gauge theories should be met with excitement and thoroughly examined. In this paper, we do this by combining two relatively recent interesting developments.

The first is the now ten-year-old observation [1] (reviewed in Ref. [2]) that compactification of large classes of four-dimensional gauge theories on a circle allows for controlled nonperturbative studies of their dynamics. The control parameter is the circle size $L$, which, when taken

\footnotetext{
*manber@1clark.edu

poppitz@physics.utoronto.ca
}

Published by the American Physical Society under the terms of the Creative Commons Attribution 4.0 International license. Further distribution of this work must maintain attribution to the author(s) and the published article's title, journal citation, and DOI. Funded by SCOAP ${ }^{3}$. small compared to the strong-coupling scale of the theory, allows for controlled studies of the ground state symmetries and spectrum. Whether the symmetry realization and spectrum change continuously in the large- $L$ limit of physical interest, however, remains a difficult question, not yet answered for general classes of theories.

The second development is the more recent discovery of novel anomaly-matching conditions, in the spirit of 't Hooft, Refs. [3,4] and references therein, but involving higher-form (here, discrete) symmetries [5,6].

In this paper, we find a novel solution to the 't Hooft anomaly-matching conditions for the nonsupersymmetric theory outlined in the abstract; more detail follows below. Remarkably, we find that the symmetry realization and massless spectrum in the calculable small- $L$ regime are identical to that of the solution of anomaly matching on $\mathbb{R}^{4}$.

While the theory we study is just one example, we hope to convince the reader that the matching is not completely trivial and that it points to the possible existence of larger classes of theories where a calculable regime - achieved by introducing a control parameter (here, $L$ ) - is continuously connected to the regime of physical interest. A deeper understanding of the correspondence between the two regimes is, clearly, very desirable.

\section{THEORY AND SYMMETRIES}

We consider $S U\left(N_{c}=2\right)$ Yang-Mills theory on $\mathbb{R}^{4}$ with $n_{f}=2$ massless adjoint Weyl fermions $\lambda$. The classical global symmetries of the theory are the continuous chiral (flavor) $S U_{f}(2)$ and axial $U(1)$. The continuous $U(1)$ axial 
group is anomalous and is broken to a $\mathbb{Z}_{4 n f}^{d \chi}$ discrete chiral symmetry, as can be seen from the action of a $U(1)$ transformation on the 't Hooft vertex due to a BelavinPolyakov-Schwarz-Tyupkin (BPST) instanton. The chiral $\mathbb{Z}_{4 n f}^{d \chi}$ is a 0 -form symmetry since it acts on local field operators. In addition, the theory can be probed by fundamental representation Wilson line operators, $W=\operatorname{tr} \exp \left[\oint_{C} a\right]$, where $a$ is the gauge field and $C$ is a noncontractible closed path (it helps to think of the theory defined on a large $\mathbb{T}^{4}$ ). Such operators transform under the $\mathbb{Z}_{2}^{C} 1$-form center symmetry. Thus, the full global (nonspace-time) symmetry of the theory is $G=S U_{f}(2) \times$ $\mathbb{Z}_{4 n f}^{d \chi} \times \mathbb{Z}_{2}^{C}$ [note that the $\mathbb{Z}_{2}$ fermion number and the center of $S U_{f}(2)$ also act as elements of $Z_{4 n f}^{d \chi}$.

\section{ANOMALY MATCHING}

Given a theory with a global symmetry $G$, it may be interesting to turn on background gauge fields of $G$. If $G$ has a 't Hooft anomaly, $G$ gauge invariance cannot be maintained. The obstruction to gauging the global symmetry, which is usually easily seen in the free UV theory, is renormalization group invariant and must be matched by the IR dynamics of the theory. This "anomaly matching" can be a powerful tool to put constraints on the theory in its strongly coupled regime.

In the following, we study the 't Hooft anomalies of the two-flavor adjoint QCD. We examine the matching in the zero-temperature phase of the theory on $\mathbb{R}^{4}$ and find a novel solution to the anomaly-matching conditions. This solution realizes the symmetries on $\mathbb{R}^{4}$ in the same way that they are known to be realized upon compactification on $\mathbb{R}^{3} \times \mathbb{S}_{L}^{1}$ at $L \Lambda_{\mathrm{QCD}} \ll 1\left(\Lambda_{\mathrm{QCD}}\right.$ is the strong-coupling scale of the theory), where the theory was solved using semiclassical methods [1].

It has been usually thought that in the nonsupersymmetric case of $n_{f}>1$ massless adjoint fermions there is a phase transition, upon increasing $L$ past $1 / \Lambda_{\mathrm{QCD}}$, associated either with the breaking of $S U\left(n_{f}\right)$ flavor symmetry, for small $n_{f}$, or with the restoration of the discrete chiral symmetry, for values of $n_{f}$ such that the theory becomes conformal ${ }^{1}$ on $\mathbb{R}^{4}$. However, as our anomaly-matching example shows, continuity between the small $\mathbb{S}_{L}^{1}$ and the $\mathbb{R}^{4}$ limits may be a feature more general than the known cases of supersymmetric YangMills theory or deformed Yang-Mills theory-where either formal arguments or a large body of evidence in favor of continuity exists.

\footnotetext{
${ }^{1}$ A conformal field theory has no scale, and hence the discrete chiral symmetry cannot be broken.
}

\section{ZERO-TEMPERATURE PHASE OF THE THEORY ON $\mathbb{R}^{4}$}

We propose that the theory is in a confined phase with unbroken $S U_{f}(2)$ and broken discrete chiral symmetry $\mathbb{Z}_{8}^{d \chi} \rightarrow \mathbb{Z}_{4}^{d \chi}$. Therefore, the theory admits two vacua which transform into each other under $\mathbb{Z}_{8}^{d x}$. In the following, we support our claim by showing that the proposed IR spectrum of the theory saturates the UV' $t$ Hooft anomalies.

In order to examine the breaking of the discrete chiral symmetry, consider the four-fermion operator, which is a singlet under the flavor $S U_{f}(2)$ and the gauge symmetry, and transforms nontrivially under $\mathbb{Z}_{8}^{d \chi}$,

$$
\mathcal{O}^{(1)} \equiv\left(\epsilon_{\alpha \beta} \lambda_{i}^{\alpha a} \lambda_{j}^{\beta a}\right)\left(\epsilon_{\alpha^{\prime} \beta^{\prime}} \lambda_{i^{\prime}}^{\alpha^{\prime} a^{\prime}} \lambda_{j^{\prime}}^{\beta^{\prime} a^{\prime}}\right) \epsilon^{i i^{\prime}} \epsilon^{j j^{\prime}}
$$

where repeated indices are summed over $[\alpha, \beta=1,2$ denote $S L(2, C)$ Lorentz indices, $i, j=1,2$ are flavor indices, while $a, b=1,2,3$ are reserved for color]. It is trivial to see that $\mathcal{O}^{(1)}$ acquires a phase $e^{i \pi}$ under $\mathbb{Z}_{8}^{d \chi}$, and hence this operator can be used to probe the breaking of this symmetry, e.g., in a lattice setup.

Our proposal for an IR behavior of the $\mathbb{R}^{4}$ theory is that at a scale of order $\Lambda_{\mathrm{QCD}}$ the four-fermion operator (1) acquires an expectation value breaking the discrete chiral symmetry, $\mathbb{Z}_{8}^{d \chi} \rightarrow \mathbb{Z}_{4}^{d \chi}$, but preserving $S U_{f}(2)$. The fermion bilinear, $\epsilon_{\alpha \beta} \lambda_{i}^{\alpha a} \lambda_{j}^{\beta a}$, usually thought responsible for the breaking of $S U_{f}(2) \rightarrow S O_{f}(2)$, is assumed to vanishas it does in the semiclassical small-circle limit.

The second part of our proposal concerns the massless spectrum of the theory. It consists of a single massless hadron, composed of three adjoint fields, with an interpolating gauge invariant local operator,

$$
\mathcal{O}_{[i j] k}^{(2) \gamma} \equiv \epsilon_{\alpha \beta} \lambda_{[i}^{\alpha a} \lambda_{j]}^{\beta b} \lambda_{k}^{\gamma c} \epsilon^{a b c}
$$

As indicated above, the operator is antisymmetric in the indices $i, j$; hence, the massless hadron (2) transforms as a fundamental under $S U_{f}(2)$ and a Weyl spinor under the Lorentz group. It also carries charge 3 under $\mathbb{Z}_{8}^{d x}$. The charges of our order parameter $\mathcal{O}^{(1)}$ and massless hadron $\mathcal{O}^{(2)}$ are summarized in Table I, in which we also list the charges of the UV adjoint fields $\lambda_{i}$. We next argue that the

TABLE I. The charges of the elementary and composite fields under the symmetries of the theory.

\begin{tabular}{lcccc}
\hline \hline & $S U_{c}(2)$ & $S U_{f}(2)$ & $\mathbb{Z}_{8}^{d \chi}$ & $\mathbb{Z}_{4}^{d \chi}$ \\
\hline$\lambda_{i}^{\alpha}$ & $\mathrm{Adj}$ & $\square$ & 1 & 1 \\
$\mathcal{O}^{(1)}$ & 1 & 1 & 4 & $4 \equiv 0$ \\
$\mathcal{O}_{[i] k}^{(2) \gamma}$ & 1 & $\square$ & 3 & 3 \\
\hline \hline
\end{tabular}


massless hadron (2) saturates the 't Hooft anomalies for all global symmetries.

We start with the only continuous 't Hooft anomaly, $\left[S U_{f}(2)\right]^{3}$. There is an odd number of $S U_{f}(2)$ fundamentals $\left(N_{c}^{2}-1=3\right)$ in the UV, and thus $S U_{f}(2)$ has a Witten anomaly. To saturate the anomaly in the IR, the theory should have an odd number of massless fermions charged under $S U_{f}(2)$. Clearly, the assumed single massless colorsinglet fermion (2) will do the trick.

The remaining nonvanishing 't Hooft anomalies all involve discrete 0 -form and 1 -form symmetries. We begin with the anomalies involving 0 -form discrete symmetries discussed some time ago in Ref. [7]. Following their classification, we consider first the more constraining "type-I" discrete anomalies $\mathbb{Z}_{4}^{d x}\left[S U_{f}(2)\right]^{2}$ and $\mathbb{Z}_{4}^{d x}[\mathcal{G}]^{2}$, where $\mathcal{G}$ denotes a background gravitational field. Notice that it suffices to consider the unbroken part of the discrete chiral symmetry (although, from Table I, this makes no difference as the charge assignments are identical; see also footnote 4).

To compute the $\mathbb{Z}_{4}^{d x}\left[S U_{f}(2)\right]^{2}$ anomaly, consider an $S U_{f}(2)$ BPST instanton background, and note that the number of fermionic zero modes is $N_{c}^{2}-1=3$, the number of $S U_{f}(2)$ fundamentals in the UV (we remind the reader that we are counting the zero modes of Weyl fermions). In the IR, the single color-singlet $S U_{f}(2)$-fundamental composite $\mathcal{O}_{[i j] k}^{(2) \gamma}$ has a a single zero mode in the background of an $S U_{f}(2)$ instanton, which carries triple the charge of an elementary adjoint Weyl fermion under $\mathbb{Z}_{4}^{d \chi}$. In effect, the IR $\mathbb{Z}_{4}^{d \chi}\left[S U_{f}(2)\right]^{2}$ anomaly gives 3 , matching the UV anomaly.

To compute the gravitational anomaly $\mathbb{Z}_{4}^{d \chi}[\mathcal{G}]^{2}$, we add up the contribution from all the flavor and color components (this counts zero modes in a gravitational instanton background; see Ref. [7]). In the UV, this gives $1 \times 2 \times 3=6$ ( 1 is the charge under $\mathbb{Z}_{4}^{d \chi}, 2$ is the number of fundamental components, and 3 is $N_{c}^{2}-1$ ). In the IR, this gives $3 \times 2 \times 1=6$, where 3 is the charge under $\mathbb{Z}_{4}^{d x}$. Again, we find an exact match between the UV and IR $\mathbb{Z}_{4}^{d x}[\mathcal{G}]^{2}$ anomaly. ${ }^{2}$

Finally, we examine the recently discovered mixed 0 form chiral/1-form center 't Hooft anomaly $\mathbb{Z}_{8}^{d \chi}\left[\mathbb{Z}_{2}^{C}\right]^{2}[5,6]$. To see the anomaly, one has to introduce a 2 -form $\mathbb{Z}_{N}^{C}$ gauge field background for the center symmetry; it suffices to consider topological backgrounds (of zero 3-form curvature). As discussed, e.g., in Ref. [8], introducing

\footnotetext{
${ }^{2}$ We note that Ref. [7] also proposed matching the less restrictive (as it is dependent on the massive spectrum) so-called type-II $\mathbb{Z}_{4}^{3}$ anomaly. It is easy to see that this condition is also obeyed here: $6 \times 1^{3}=2 \times 3^{3}(\bmod m 4)$, where $m \in \mathbb{Z}$, as per Ref. [7].
}

topological $\mathbb{Z}_{2}^{C} 2$-form background gauge fields is equivalent $^{3}$ to allowing nontrivial 't Hooft fluxes [10] when considering the theory on $\mathbb{T}^{4}$.

Now, under a discrete $\mathbb{Z}_{8}^{d \chi}$ transformation, the fermion measure transforms by a phase factor $e^{i 2 \pi Q}$. Here, $Q=$ $\frac{\int \operatorname{Tr} F \wedge F}{8 \pi^{2}}$ is integer when the 't Hooft flux vanishes; hence, $\mathbb{Z}_{8}^{d x}$ is anomaly free in the theory without centerbackground gauge fields. However, $Q=\frac{n_{12} n_{34}}{N_{c}}+n$, $n \in \mathbb{Z}$, when, e.g., only the 't Hooft fluxes $n_{12}$ and $n_{34}$ (which are integers modulo $N_{c}$ ) are nonvanishing. Thus, for our two-color case, taking $n_{12}=n_{34}=1$, the partition function acquires a phase $e^{i \pi}$ under $\mathbb{Z}_{8}^{d \chi}$ transformations. This phase is the manifestation of the mixed discrete-chiral/ center-squared anomaly. It is independent of the $\mathbb{T}^{4}$ volume and can be argued to be renormalization group invariant; e.g., it can be seen to be due to the variation of a fivedimensional local bulk term involving only $\mathbb{Z}_{8}^{d \chi}$ and $\mathbb{Z}_{2}^{C}$ background fields $[5,6,8,11]$.

In the IR theory, this anomaly is matched ${ }^{4}$ in the "Goldstone" (spontaneously broken) mode due to the assumed nonvanishing $\mathcal{O}^{(1)}$ vacuum expectation value. $\mathrm{A} \mathbb{Z}_{8}^{d \chi}$ symmetry transformation interchanges the two vacua and, in the background of 't Hooft fluxes, transforms the partition function by a $e^{i \pi}$ phase, as in Refs. $[5,6]$.

As usual, anomaly-matching arguments do not determine the spectrum of massive states associated with the discrete symmetry breaking. However, the recent insights of Refs. $[5,6,8,11]$ shed some light on the properties of the domain walls between the two vacua. For example, using a discrete version of anomaly inflow [6], one can argue that the center symmetry-assumed or shown to be unbroken in the bulk; see below-is broken on the domain walls and the fundamental Wilson loop has a perimeter law there. This has been explicitly seen in the semiclassical regime on $\mathbb{R}^{3} \times \mathbb{S}^{1}$ [13] and also argued in Ref. [8]. It would be interesting to further study the dynamics of the various

\footnotetext{
${ }^{3}$ This becomes especially clear-and is well known-on the lattice, where the simplest $\mathbb{Z}_{N}^{C}$ 2-form gauge field topological background is the one corresponding to the insertion of a thin center vortex (corresponding, e.g., to a $n_{12}$ 't Hooft flux in the continuum; see Ch. 4 of Ref. [9] and references therein).

${ }^{4}$ The observant reader may notice that the massless fermion (2) also matches all purely 0 -form anomalies if the unbroken $\mathbb{Z}_{4}^{d x}$ chiral symmetry is replaced by the full $\mathbb{Z}_{8}^{d \chi}$. However, an IR theory of a single massless gauge-singlet fermion cannot match the mixed 0-form/1-form discrete anomaly discussed here; it would not couple to 't Hooft flux and could not give rise to the mixed chiral/center anomaly, unless there were other massless (or topological) degrees of freedom (d.o.f.) present. Without such d.o.f., the $\mathbb{Z}_{8}^{d \chi} \rightarrow \mathbb{Z}_{4}^{d \chi}$ breaking appears as the only consistent possibility (see, however, Ref. [12] for a scenario with unbroken $\mathbb{Z}_{8}^{d \chi}$ and an emergent massless gauge field).
} 
domain walls in this theory, in the zero and finite-temperature cases on both $\mathbb{R}^{3} \times \mathbb{S}^{1}$ and $\mathbb{R}^{4}[8,14,15]$.

In summary, the 't Hooft matching conditions of $S U_{c}(2)$ Yang-Mills with two adjoint Weyl flavors allow for a phase with a massless composite fermion in the IR. The theory preserves its $S U_{f}(2)$ chiral symmetry and has two vacua that preserve a $\mathbb{Z}_{4}^{d x}$ subgroup of the $\mathbb{Z}_{8}^{d \chi}$ discrete chiral symmetry.

We also expect the theory to preserve its $\mathbb{Z}_{2}^{C} 1$-form symmetry and hence the fundamental Wilson loop to obey an area law. The area-law expectation is consistent with the observed behavior as we compactify the theory on a circle $\mathbb{S}_{L}^{1}$ of circumference $L$ (where the fermions obey periodic boundary conditions along $\mathbb{S}_{L}^{1}$ ) and interpolate between $L \Lambda_{\mathrm{QCD}} \ll 1$ and $L \Lambda_{\mathrm{QCD}} \gg 1$. In the small-circle limit, one can integrate an infinite tower of Kaluza-Klein excitations of the gauge field and fermions along $\mathbb{S}_{L}^{1}$, which generates an effective potential for the holonomy $\Phi \equiv \oint_{\mathbb{S}_{L}^{1}} A$. This potential is minimized at a nonzero value of $\Phi$, which breaks $S U(2)$ down to $U(1)$. Thus, the theory Abelianizes, enters its weakly coupled regime (since $L \Lambda_{\mathrm{QCD}} \ll 1$ ), and becomes amenable to semiclassical treatment. The Abelian field can be taken along the $\tau^{3}$-direction (the Cartan component) in the color space, and thus the adjoint fermions in this direction stay massless and uncharged ${ }^{5}$ under $U(1)$. Then, the effective three-dimensional $U(1)$ theory can be dualized to a free compact scalar $\sigma$. The story does not stop here since this theory admits magnetic bions [1], which are non-self-dual nonperturbative saddles. The proliferation of magnetic bions leads to the generation of a potential, $V(\sigma)=\cos 2 \sigma$; see Ref. [1] for more details. Thus, the IR theory has two vacua at $\sigma=0$ and $\sigma=\pi$, and hence the discrete chiral symmetry $\mathbb{Z}_{8}^{d \chi}$ breaks to $\mathbb{Z}_{4}^{d \chi}$. This theory has a mass gap and confines the fundamental electric charges [1]. In addition, as indicated above, there is an $S U_{f}(2)$-doublet massless excitation which is neutral under $U(1)$ : the Cartan component of the adjoint fermions. Notice that this is the same number of massless fermions as in our proposed phase on $\mathbb{R}^{4}$. Therefore, the theory in the semiclassical regime on $\mathbb{R}^{3} \times \mathbb{S}^{1}$ shares all the features of the proposed phase of the theory on $\mathbb{R}^{4}$, and we expect the continuity of the mass gap from the small to the large circle to hold as well.

\section{DISCUSSION AND RELATION TO OTHER STUDIES}

To recuperate our proposed spectrum and symmetries, we argue that in the $S U_{c}(2)$ theory with $n_{f}=2$ massless Weyl adjoint fermions the $S U_{f}(2)$ is unbroken, the theory has two vacua due to $\mathbb{Z}_{8}^{d \chi} \rightarrow \mathbb{Z}_{4}^{d \chi}$ symmetry breaking, and

\footnotetext{
${ }^{5}$ This can be easily seen from the covariant derivative of the adjoint fermions.
}

the IR spectrum consists of a single massless $S U_{f}(2)$ doublet Weyl fermion with the quantum numbers of Table I.

For the theory at hand, we find it remarkable that the anomaly-matching solution on $\mathbb{R}^{4}$ realizes the IR spectrum and symmetries in a manner similar to the one found by semiclassically solving the theory on $\mathbb{R}^{3} \times \mathbb{S}^{1}$.

A question that may lurk in the reader's mind is whether such a continuity of the symmetry realization between small and large $\mathbb{S}^{1}$ is consistent with anomalies also in higher rank and/or larger- $n_{f}$ adjoint QCD theories. While we cannot prove or disprove the existence of solutions of anomaly matching for a larger class of adjoint QCD theories, they are not trivial to construct. The case we discuss here is likely the simplest, and a devoted search for others is left for the future.

As usual, any solution of 't Hooft's anomaly matching comes with a caveat-it only gives a possible consistent IR behavior of the theory, albeit one which may or may not be dynamically realized. Clearly, to rule in or out our proposed realization of the symmetries, further analytical and numerical studies are needed.

On the numerical side, the $S U_{c}(2)$ gauge theory with a single Dirac flavor $\left(n_{f}=2\right.$ Weyl) in the adjoint has been the subject of the lattice studies [16,17], which found that the IR behavior of the theory appears inconsistent with the conventional continuous $S U_{f}(2) \rightarrow S O_{f}(2)$ chiral symmetry breaking, pointing "tentatively" (the characterization is from Ref. [16]) toward conformal or near-conformal behavior. The authors considered only the two hitherto known possibilities consistent with anomaly matching: conformal behavior or continuous chiral symmetry breaking. The IR scenario we propose here offers a third way but naturally poses challenges for lattice studies; the theory is, indeed, conformal in the deep IR, with the massless fermion the only relevant d.o.f., but with a likely complicated massive spectrum associated with the discrete symmetry breaking. The fermions are described by the Ginsparg-Wilson action with a bare mass (or hopping parameter), which will have the correct discrete chiral symmetry as well as the 0 -form and 1-form mixed anomaly in the chiral limit. One can then study the histogram of the operator $\mathcal{O}^{(1)}$ as a function of the hopping parameter. In our scenario, the real part of this operator should fluctuate between two values in the continuum limit, indicating the breaking of $\mathbb{Z}_{8}^{d x}$ to $\mathbb{Z}_{4}^{d x}$. Some progress along this line of thought in supersymmetric lattice models was achieved in Ref. [18].

On the analytical side, the beta function of Yang-Mills theory with arbitrary representations was recently computed to four loops [19]. While the results are scheme dependent, it is amusing to note some interesting features of the beta function. Adapting Ref. [19] to the $n_{f}=2$ Weyl adjoint $S U_{c}(2)$ theory, one finds, first, the well-known 
result that the two-loop beta function shows no IR fixed point. Second, one finds that IR fixed points appear at three and four loops, with the value of the scheme-dependent fixed-point coupling becoming smaller as the number of loops is increased from three to four (we stress again that it is not obvious to us what these perturbative calculations teach us about the IR behavior of the theory). Another amusing exercise is to redo this for other values of $n_{f}$. For $n_{f}=1\left[S U_{c}(2)\right.$ super-Yang-Mills], one finds no IR fixed points up to the four loops of Ref. [19], while for $n_{f} \geq 3$, a fixed point appears already at two loops (the fixed-point coupling moves to weaker values as the number of loops is increased from two to four).

To rule in or out conformality - or our proposal—one should further improve the lattice studies of [16] and study other indications of conformality. These indications include: (i) the running coupling on the lattice in the $n_{f}=$ 2 theory, as done for $n_{f}=4$ in [20] (we note that, recently, [21] found no IR fixed point for $n_{f}=2$ in the range of masses studied), (ii) the area vs perimeter law for the Wilson loop, as advocated in [22], and (iii) the expected nontrivial properties of domain walls, which should be present in any phase with broken chiral symmetry.

\section{ACKNOWLEDGMENTS}

We thank Ken Intriligator and Julius Kuti for discussions relevant to this paper. We are also indebted to Thomas Dumitrescu for patiently explaining to us the results of Ref. [12]. M. A. is supported by NSF Grant No. PHY1720135 and the Murdock Charitable Trust. E.P. is supported by a Discovery Grant from NSERC.

Note added.-Recently, Ref. [12] appeared, showing that formulating our theory on a nonspin manifold gives rise to new 't Hooft anomalies, absent on spin manifolds (nonetheless, these anomalies impose constraints on the flat space theories). A consequence of these new anomalies is that our scenario for the IR physics of two-flavor adjoint QCD should be supplemented with an emergent $\mathbb{Z}_{2}$ gauge theory with a broken (also emergent) $\mathbb{Z}_{2} 1$-form global symmetry, which can be probed by appropriate 't Hooft loop operators; see Ref. [12].
[1] M. Unsal, Phys. Rev. D 80, 065001 (2009).

[2] G. V. Dunne and M. Unsal, Annu. Rev. Nucl. Part. Sci. 66, 245 (2016).

[3] Y. Frishman, A. Schwimmer, T. Banks, and S. Yankielowicz, Nucl. Phys. B177, 157 (1981).

[4] S. R. Coleman and B. Grossman, Nucl. Phys. B203, 205 (1982).

[5] D. Gaiotto, A. Kapustin, N. Seiberg, and B. Willett, J. High Energy Phys. 02 (2015) 172.

[6] D. Gaiotto, A. Kapustin, Z. Komargodski, and N. Seiberg, J. High Energy Phys. 05 (2017) 091.

[7] C. Csaki and H. Murayama, Nucl. Phys. B515, 114 (1998).

[8] Z. Komargodski, T. Sulejmanpasic, and M. Unsal, Phys. Rev. B 97, 054418 (2018).

[9] J. Greensite, Lect. Notes Phys. 821, 1 (2011).

[10] G. 't Hooft, Nucl. Phys. B153, 141 (1979).

[11] Z. Komargodski, A. Sharon, R. Thorngren, and X. Zhou, arXiv:1705.04786.

[12] C. Cordova and T. T. Dumitrescu, arXiv:1806.09592.
[13] M. M. Anber, E. Poppitz, and T. Sulejmanpasic, Phys. Rev. D 92, 021701 (2015).

[14] H. Shimizu and K. Yonekura, Phys. Rev. D 97, 105011 (2018).

[15] M. M. Anber, E. Poppitz, and M. Unsal, J. High Energy Phys. 04 (2012) 040.

[16] A. Athenodorou, E. Bennett, G. Bergner, and B. Lucini, Phys. Rev. D 91, 114508 (2015).

[17] G. Bergner, P. Giudice, G. Mnster, P. Scior, I. Montvay, and S. Piemonte, J. High Energy Phys. 01 (2018) 119.

[18] S. Ali, G. Bergner, H. Gerber, P. Giudice, I. Montvay, G. Mnster, S. Piemonte, and P. Scior, J. High Energy Phys. 03 (2018) 113).

[19] M. F. Zoller, J. High Energy Phys. 10 (2016) 118.

[20] J. Rantaharju, T. Rantalaiho, K. Rummukainen, and K. Tuominen, Phys. Rev. D 93, 094509 (2016).

[21] G. Bergner and S. Piemonte, Phys. Rev. D 97, 074510 (2018).

[22] E. Poppitz and M. Unsal, J. High Energy Phys. 09 (2009) 050. 